Rapport - Société canadienne d'histoire de l'Église catholique

\title{
L'éducation au temps de Mgr de Laval
}

\section{Louis-Philippe Audet}

Volume 25, 1957-1958

URI : https://id.erudit.org/iderudit/1007443ar

DOI : https://doi.org/10.7202/1007443ar

Aller au sommaire du numéro

Éditeur(s)

La Société canadienne d'histoire de l'Église catholique

ISSN

0318-6148 (imprimé)

1927-7075 (numérique)

Découvrir la revue

Citer cet article

Audet, L.-P. (1957). L'éducation au temps de Mgr de Laval. Rapport - Société canadienne d'histoire de l'Église catholique, 25, 59-78.

https://doi.org/10.7202/1007443ar

Tous droits réservés @ La Société canadienne d'histoire de l'Église catholique, 1958
Ce document est protégé par la loi sur le droit d'auteur. L’utilisation des services d'Érudit (y compris la reproduction) est assujettie à sa politique d'utilisation que vous pouvez consulter en ligne.

https://apropos.erudit.org/fr/usagers/politique-dutilisation/ 


\section{L'éducation au temps de Mgr de Laval ${ }^{1}$}

Le 8 décembre 1958 marquera le troisième centenaire de la consécration de $\mathrm{M}^{\mathrm{gr}}$ François de Montmorency Laval comme évêque de Pétrée in partibus infidelium et vicaire apostolique de toute la Nouvelle-France. Et l'année prochaine, celle de 1959, nous rappellera également d'autres anniversaires importants, notamment celui de l'arrivée à Québec, le 16 juin 1659, du fondateur de l'Eglise canadienne. Tous ces motifs ont incité sans doute la Société canadienne d'Histoire de l'Eglise catholique à centrer la plupart des travaux de ce Congrès sur la haute personnalité de $\mathbf{M}^{\mathrm{gr}^{\mathrm{r}}}$ de Laval. Il convient de l'en féliciter.

Rappelons encore une fois que $\mathrm{M}^{\mathrm{gr}}$ de Laval est né le 30 avril 1623 , qu'il fut choisi comme premier évêque de Québec en mai 1658, qu'il eut comme premier successeur $\mathrm{M}^{\mathrm{gr}}$ de Saint-Vallier en 1684, qu'il dut continuer d'administrer son vaste diocèse durant les douze années que celui-ci passa en France, qu'il mourut enfin à Québec le 6 mai 1708, à l'âge de 85 ans. Il était évêque depuis cinquante ans et il avait gouverné de droit ou de fait l'Eglise du Canada pendant 35 ans.

L'une des tâches les plus importantes de l'Evêque, c'est de veiller sur l'éducation de ses ouailles. Il me semble donc tout indiqué de nous demander ici ce que fut:
I. - l'éducation en France au XVII ${ }^{\bullet}$ siècle ?
II. - l'éducation en Nouvelle-France de 1635 à 1700 ?
III. - l'action de $\mathrm{M}^{\mathrm{gr}}$ de Laval dans ce domaine?

\section{I. - L'ÉDUCATION EN FRANCE AU XVIIe SIÈCLE.}

Pour bien comprendre ce qui s'est accompli sur les bords du SaintLaurent, dans le domaine de l'éducation, pendant la seconde moitié du $\mathrm{XVII}^{\circ}$ siècle, il est tout à fait essentiel de connaître quel était l'état de l'éducation en France à cette époque. Car les premiers missionnaires et les premiers évêques inclineront naturellement à doter le Canada d'institutions scolaires identiques à celles dont ils furent les élèves ou même les maîtres outre-Atlantique.

Rappelons d'abord que la pédagogie française du XVII siècle est caractérisée par trois courants principaux: le courant catholique, le courant janséniste et le courant protestant.

1 Travail présenté au $X X X V^{e}$ Congrès de la Société canadienne d'Histoire de l'Eglise catholique, le 11 octobre 1958, à Québec. 
Parmi les grands noms de la pédagogie catholique, il convient de mentionner Descartes, Bossuet, Fénelon, les Oratoriens, saint JeanBaptiste de la Salle, madame de Maintenon, etc.: les traditions chrétiennes, les Pères de l'Eglise, le moyen âge et la Renaissance servent d'inspiration aux grandes lignes de cette pédagogie qui va exercer une influence considérable.

Le courant janséniste représenté surtout par les écoles de PortRoyal-des-Champs est caractérisé par un rigorisme en opposition avec le véritable esprit du christianisme qui est un esprit de douceur et de bonté; cette pédagogie va à l'encontre des données de la psychologie de l'enfant. Il faut convenir, d'autre part, que le mérite de Port-Royal fut de comprendre la nécessité de l'éducation à une époque où cet important devoir était souvent négligé; mais ces pédagogues ne surent pas garder la mesure dans une réforme excellente en soi et même nécessaire.

Bacon, Ratichius, Comenius, Francke et Locke furent les principaux protagonistes du courant pédagogique protestant au $\mathrm{XVII}^{e}$ siècle: ils s'efforcent de réagir contre un humanisme exagéré qui, à leur sens, ne préparait pas assez à la vie pratique. La science, selon eux, devait avoir un rôle très considérable à jouer dans l'éducation de l'enfant ${ }^{2}$.

Dans le secteur de l'enseignement ELEMENTAIRE, je crois nécessaire de rappeler ici quelques noms célèbres: leur influence, en France, fut considérable et il est bien évident que nos premiers éducateurs furent en quelque sorte les héritiers spirituels de ces pédagogues catholiques.

Et tout d'abord, le lorrain PIERRE FOURIER, curé de Mattaincourt, qui fonda la Congrégation de Notre-Dame pour l'éducation des filles; né en 1565, il mourut en 1640. L'une de ses filles spirituelles, Marguerite Bourgeoys, viendra au Canada pour y commencer une œuvre identique dont nous connaissons tout le rayonnement. Saint Pierre Fourier fut le premier à fonder une communauté de femmes vouées spécialement à l'instruction et à l'éducation des jeunes filles ${ }^{3}$.

L'abbé CHARLES DEMIA exerça son zèle dans la région lyonnaise. En 1666, il publie un livre intitulé Remontrances qui n'est qu'un vigoureux plaidoyer en faveur de l'éducation populaire. En 1671, il ouvrait une première école; cette fondation fut suivie de plusieurs autres dont il assuma la direction. Il s'occupa activement de la formation des maîtres d'école et fonda même la communauté des Sœurs de Saint-Charles destinée à l'enseignement du peuple ${ }^{4}$.

Mais le plus illustre nom de la pédagogie française au XVII ${ }^{e}$ siècle, c'est celui de JEAN-BAPTISTE DE LA SALLE, le fondateur des Frères des Ecoles chrétiennes: né à Reims en 1651, il mourut à Rouen en 1719. En raison de ces dates, on comprendra que l'influence lasallienne au Canada, de 1635 à 1700 , fut à peu près nulle.

2 L. Riboulet, Histoire de la Pédagogie, Emmanuel Vitte, Paris, 1942, 667 pp.

3 Renaud, Les idées pédagogiques de saint Pierre Fourier, Paris, Lethiellieux. Bernard, Lettres choisies de saint Pierre Fourier, Paris, 1920

4 Compayré, Charles Démia - coll. Les grands éducateurs: Vie de Charles Démia, Lyon, 1829. 
L'abbé Démia, dont je viens de parler, nous a laissé une vivante peinture de la physionomie de ces écoles populaires ou "petites écoles ", comme on les désignait alors. Dans son ouvrage intitulé L'Ecole paroissiale ou la manière de bien instruire les enfants des petites écoles ${ }^{5}$ il en précise le programme, en décrit les manuels et les méthodes à utiliser.

Le programme de ces petites écoles françaises, au milieu du XVII ${ }^{\circ}$ siècle est des plus simples: on s'occupait alors beaucoup plus de l'éducation que de l'instruction. L'auteur divise son travail en trois parties. Dans la première, il signale les qualités du maître d'école, les conditions d'une bonne organisation matérielle et les exigences pour l'admission des élèves. $\mathrm{La}$ seconde partie est consacrée à la méthodologie de l'enseignement religieux et la troisième à la méthodologie des autres spécialités du programme.

L'enseignement religieux reçoit une attention particulière; puis vient la lecture qui revêt une importance de premier ordre. L'enfant apprend d'abord à lire le latin avant le français. Les petites écoles enseignent les lettres, puis l'épellation et la lecture d'ouvrages latins. Lorsque l'enfant se débrouille convenablement dans cette tâche, il aborde la lecture des livres français qui sont gradués selon son âge et son degré d'avancement, le "summum" du savoir étant La Civilité chrétienne. Il ne lui restait plus alors qu'à apprendre à lire des lettres écrites à la main. "La lecture des papiers ou des manuscrits, note un historien de cette époque, complétait l'enseignement de la lecture: on allait chercher dans les greniers et au fond des armoires de vieux registres et des contrats poudreux, écrits souvent en caractères impossibles à lire, et quand l'élève parvenait à les déchiffrer convenablement, le maître n'avait plus rien à lui apprendre ${ }^{6}$." Cet enseignement de la lecture était complété par des notions sommaires d'orthographe. Rien de très compliqué cependant: on y discute de la façon d'écrire certains mots et l'on donne aux élèves à copier quelques lignes de la leçon. Et voilà pour l'enseignement du français.

L'écriture figure également en place d'honneur dans le programme des petites écoles. L'auteur de $L^{\prime} E$ cole paroissiale donne sur le sujet des conseils fort pertinents, il entre dans tous les détails propres à assurer l'efficacité de cet enseignement: exemples habiles imprimés ou à la main, manière de former les lettres,' de les lier ensemble, façon de tenir la plume, etc. Le bagage de connaissances se complète enfin par des notions de calcul, auxquelles on ajoutait parfois les éléments du latin pour ceux qui manifestaient des dispositions particulières pour les études.

Tel était le programme des écoles primaires en France au XVII ${ }^{\circ}$ et même au $\mathrm{XVIII}^{\circ}$ siècles: L'Ecole paroissiale n'exigeait pas autre chose.

Des causes de divers ordres favorisèrent l'épanouissement et la diffusion de L'ENSEIGNEMENT SECONDAIRE et même SUPERIEUR

5 Ouvrage édité par Pierre Targa, Paris, 1654.

6 Allain, L'enseignement primaire en France avant la Révolution, p. 168. Librairie de la Société bibliographique, 35, rue de Grenelle, Paris, 1881. 
en France durant le XVII ${ }^{e}$ siècle: multiplication des séminaires confiés à des Sociétés ou Congrégations religieuses (Oratoriens, Lazaristes, Sulpiciens, Eudistes, etc.), fondation de l'Académie française, des Académies nationales et des sociétés savantes de province, sans parler des Collèges dirigés par les Jésuites et dont l'influence sera capitale sur l'organisation et l'orientation des études au Collège de Québec. Au demeurant, l'éducation secondaire et supérieure du $\mathrm{XVII}^{\mathrm{e}}$ siècle est tout imprégnée des idées pédagogiques de Descartes, de Bossuet, de Fénelon, des Oratoriens, des Jésuites et des Messieurs de Port-Royal.

La réforme de l'Université par Henri IV date de 1598. Le latin est alors la langue des gens cultivés, la langue parlée depuis quinze cents ans, c'est aussi la langue de l'Eglise: l'enseignement du latin prendra donc la prépondérance dans le programme des Humanités. "L'écolier, écrit Claude Galarneau, doit apprendre le latin pour le parler et l'écrire. Le latin est même la langue obligatoire de la conversation et la langue par laquelle on apprend toutes les autres matières. Quatre ans sont consacrés à la grammaire, aux principes et aux règles. C'est plus que la grammaire puisque la poétique et la rhétorique entrent dans ce que les gens du XVII ${ }^{e}$ siècle appellent la grammaire ${ }^{7}$.»

L'organisation des Collèges jésuites est quelque peu différente: on y enseigne le latin avec des méthodes nouvelles. Le plan des études est déterminé par la règle vingt et unième du Père provincial: "Il ne doit pas y avoir plus de cinq classes dans un collège secondaire: une de Rhétorique, une d'Humanité, trois de Grammaire... ${ }^{8}$." Le programme comporte l'enseignement du latin et du grec. Cet enseignement, il va sans dire, se fait en latin (les premières grammaires latines françaises n'apparaîtront qu'à la fin du XVII ${ }^{\text {e }}$ siècle); quant au grec, il sera inscrit au programme afin de rivaliser avec les collèges fondés par les protestants et aussi pour permettre aux élèves de connaître les sources grecques de la religion ${ }^{9}$.

Un programme de mathématiques pour les trois années de philosophie sera ensuite préparé et sera en vigueur dans tous les Collèges de la Compagnie de Jésus, dès 1597. Il convient de noter ici que les mathématiques sont réservées au cycle supérieur des études car les Pères considèrent la formation par les sciences comme un humanisme pour adultes ${ }^{10}$; d'ailleurs, parmi les élèves du cycle supérieur, un tout petit nombre seulement $(7 \%)$ s'orientera du côté scientifique.

La pédagogie jésuitique ne fut pas adoptée par toutes les maisons d'enseignement secondaire: c'est ainsi que les maîtres de Port-Royal préconisent l'enseignement du latin par le français et pour le français, avec des manuels rédigés en français; les Pères de l'Oratoire formulent

7 Claude Galarneau, L'enseignement des Humanités au XVI et au XVII siècles, Mémoire présenté à la Commission du Programme, Faculté des Arts, Université Laval, 26 mars 1958; CP $57 \mathrm{~d}$.

8 Florian Larivière, s.j., Notes sur la pédagogie des Jésuites, Commission du Programme, Faculté des Arts, Université Laval; CPS (2) b.

9 François de Dainville, La Naissance de l'Humanisme moderne, p. 46, Paris, 1940.

10 François de Dainville, op. cit., p. 66. 
des critiques identiques et marchent sur les brisées de Port-Royal dans ce domaine. Malgré ces critiques et ces tentatives de réforme, les Jésuites et les Collèges de l'Université continuent à tout enseigner en latin et à accorder la primauté au thème ${ }^{11}$. Et ce ne sera qu'après 1715 qu'on suivra l'exemple de Port-Royal et de l'Oratoire et encore avec ménagement: le Père de Jouvency prendra soin d'avertir dans son Ratio docendi (1711) « qu'il faut prendre garde qu'on ne passionne les jeunes pour leur langue maternelle qui fait perdre le temps et la moralité ${ }^{12}$ ». C'est pourquoi il borne cet enseignement du français aux classes de grammaire.

Dans ce programme des études secondaires, c'est le professeur de grammaire ou de rhétorique qui enseigne la géographie et l'histoire; de même les professeurs de philosophie seront-ils chargés des cours de science. Cet enseignement est donné en latin et à l'intérieur du cours de philosophie. Les sciences mathématiques et physiques font bien des progrès extraordinaires avec Galilée, Descartes, Pascal, Newton: malheureusement, il manque des vulgarisateurs et c'est le XVIII ${ }^{\circ}$ siècle qui les produira bientôt.

Voilà dans ses grandes lignes quelle fut l'organisation de l'enseignement primaire et secondaire en France au XVII ${ }^{e}$ siècle. Dans quelle mesure, les premiers missionnaires vont-ils réussir à reconstituer des cadres identiques dans la colonie naissante, au palier de l'enseignement élémentaire aussi bien que dans le domaine de l'enseignement secondaire? C'est ce qu'il faut maintenant fixer.

\section{II. - L'ÉDUCATION EN NOUVELLE-FRANCE DE 1635 A 1700.}

Sous le régime français, l'enseignement primaire fut dispensé par ces institutions que l'on nommait alors, au Canada comme en Europe, les petites écoles. Le programme était celui que l'on retrouve dans ce traité de pédagogie dont nous avons parlé tout à l'heure, L'Ecole paroissiale, ouvrage qui connut beaucoup de vogue au $\mathrm{XVII}^{\circ}$ et au $\mathrm{XVIII}^{\circ}$ siècles. Ce programme n'est pas compliqué: aux petits Canadiens, comme aux petits Français, l'on enseignait le catéchisme, la lecture, l'écriture et le calcul. Les mieux doués apprenaient les rudiments du latin afin de se préparer aux études secondaires. Le programme ne varie guère pour les filles, sinon qu'il se complète par des notions d'enseignement ménager ${ }^{12 \mathrm{a}}$.

" Apprendre aux enfants à lire, à écrire et à compter, leur enseigner le catéchisme, les former à la vertu, voilà ce que voulaient d'abord, en fondant des écoles, $\mathbf{M}^{\mathrm{gr}}$ de Laval, $\mathbf{M}^{\mathrm{gr}}$ de Saint-Vallier, les Pères Jésuites,

11 Claude Galarneau, op. cit., CP 57 e.

12. Claude Galarneau, op. cit., CP $57 \mathrm{~g}$.

12a $M^{\mathrm{gr}}$ de Laval était en France lorsqu'un mandement de l'archevêque de Lyon, le $1^{\text {er }}$ février 1675 , avait donné à l'abbé Démia e la conduite et la direction des petites écoles du diocèse ". $M^{\mathrm{gr}}$ de Laval se fit envoyer à Québec la Méthode pour faire les Ecoles et le Règlement. Cf. Auguste Gosselin, Vie de $\mathrm{M}^{\mathrm{gr}}$ de Laval, 1890, I: 583. 
les Sulpiciens, les Frères Charron, etc. Pas d'histoire, pas de géographie, très peu de grammaire dans les petites écoles ${ }^{13}$."

Les écoles de filles et de garçons suivaient sensiblement le même programme, sauf que, pour les premières, on ajoutait au catéchisme, à la lecture et au calcul, " toutes sortes d'ouvrages propres à leur sexe», dira Marie de l'Incarnation. $\mathbf{M}^{\mathrm{gr}}$ de Laval, dans son approbation des Sours de la Congrégation de Notre-Dame, soulignera que les religieuses a élevaient les petites filles dans la crainte de Dieu et l'exercice des vertus chrétiennes, leur apprenant à lire et à écrire et les autres travaux dont elles sont capables ".

Nous pouvons donc conclure que les programmes et les méthodes d'enseignement furent à peu près identiques dans les petites écoles du Canada et dans celles de France. Le but n'était pas de faire des savants: l'histoire nous apprend que ces institutions formèrent des hommes et des femmes de cour et d'énergie, qu'elles contribuèrent à répandre parmi les colons une grande affabilité, des manières douces et polies qui frappèrent souverainement les voyageurs et les historiens qui visitèrent la Nouvelle-France à cette époque.

Mais quels manuels utilisait-on alors ? Voilà une autre question qui ne manque pas d'intérêt, surtout lorsqu'on se rappelle qu'il n'y eut pas d'imprimerie au Canada sous le régime français. On fut donc obligé d'importer les manuels scolaires.

Dans ces commandes de livres, on relève d'abord la présence d'ouvrages de pédagogie générale ou mieux de méthodologie, tels l'Ecole paroissiale de l'abbé Charles Démia, Le Règlement pour les maîtres d'école du diocèse de Lyon et La Méthode pour faire les Ecoles. Quant aux livres utilisés pour les petites écoles, ils sont tous empruntés à la France: le Petit Alphabet, le Grand Alphabet, le Psautier, les Pensées chrétiennes, l'Introduction à la Vie dévote, le Pédagogue, la Civilité chrétienne, les Manuscrits et Contrats, la Bienséance et civilité chrétienne, l'Instruction de la Jeunesse, le Petit Office de Notre-Dame, le Nouveau Testament, le Catéchisme, le Syllabaire, etc.

Mais ces petites écoles furent-elles bien nombreuses durant le XVII ${ }^{\bullet}$ siècle, c'est-à-dire durant les soixante-quinze premières années de la colonie ? Distinguons entre les écoles des grands centres (Québec, Trois-Rivières et Montréal) et les écoles de campagne.

Dès 1635 nous voyons les Jésuites inaugurer l'enseignement primaire à Québec; en 1699, le Séminaire de Québec ouvrait une école à la Hauteville et, à une date difficile à déterminer, la Basse-ville était aussi dotée d'une institution semblable. Les filles, pour leur part, pouvaient s'inscrire, dès 1639, chez les Ursulines et, à partir de 1686, au couvent des Sours de la Congrégation. Plus tard, en 1725, les Sœurs de l'Hôpital Général ajouteront, au soin des malades, la tenue d'un pensionnat.

$\mathrm{La}$ ville et la région des Trois-Rivières furent relativement peu peuplées durant le régime français: il est évident, toutefois, que les

$13 \quad \mathrm{M}^{\mathrm{gr}}$ Amédée Gosselin, L'instruction au Canada sous le régime français, p. 236. 
Récollets tinrent école pendant le temps où ils furent en charge de la cure, soit de 1671 à 1683 et de 1693 à 1777 . Il est incontestable, d'autre part, que trois instituteurs laïques tiennent école au bénéfice de la jeunesse trifluvienne: il s'agit d'abord de Séverin Ameau, notaire, chantre et pédagogue, vers 1652. Son successeur fut peut-être Jean-Baptiste Pothier, également notaire et qui arrive aux Trois-Rivières en 1700 après avoir enseigné à Lachine dès 1686. Enfin voici le sacristain Rigault à qui le vicaire général, $M$. de Miniac recommande de faire payer la somme promise parce qu'il a tenu école ${ }^{14}$. Les filles, de leur côté, peuvent bénéficier de l'enseignement des Sœurs de la Congrégation, à partir de 1664, et de celui des Ursulines dès 1697.

Montréal n'eut rien à envier, sous le rapport de l'instruction primaire, aux deux autres centres. Une petite école fut ouverte pour les garçons en 1666, par les soins de l'abbé Souart, supérieur de SaintSulpice et ancien curé de Notre-Dame, qui tint lui-même à en être le premier instituteur. Vingt ans plus tard, en 1686, une "Association de citoyens de Ville-Marie pour les Ecoles de cette ville " était formée par l'initiative de Mathurin Rouiller, Nicolas Barbier, Philibert Boy et Jacob Thomelet. Etablie rue Notre-Dame, face au Séminaire, cette petite école dispensa l'enseignement élémentaire durant sept ans. Le 9 octobre 1693, les Messieurs de Saint-Sulpice en héritaient par suite de la disparition de deux membres de l'Association. Signalons ici le zèle des Frères Charron qui s'intéressèrent à l'éducation de la jeunesse dès 1694. Cependant, c'est aux Søurs de la Congrégation de Notre-Dame que revient l'honneur d'avoir établi la première école à Ville-Marie, en 1657. Durant les neuf premières années de la fondation, Marguerite Bourgeoys accueillit les garçons et les filles; à partir de 1666, elle se contenta de dispenser l'enseignement aux filles.

En ces heures difficiles du début de la colonie, il est bien évident que les campagnes ont été moins bien pourvues d'écoles que les grands centres. Les petites écoles que l'on voit surgir avant 1700 dans la région de Québec sont établies à St-Joachim (avant 1676), à Sainte-Foy (avant 1673), au Château-Richer (avant 1674), à l'Ile d'Orléans (avant 1676) et à Saint-Joseph de Lévy en 1694. D'autre part, les Sœurs de la Congrégation commencent à essaimer et on les voit ouvrir des couvents de campagne à Champlain vers 1680 , à Pointe-aux-Trembles, près de Montréal en 1680, à Sainte-Famille de l'Ile d'Orléans en 1685, à Lachine en 1686, au Château-Richer en 1694, à Boucherville en 1703 et à La Prairie de la Madeleine en 1703 également.

A l'enseignement dispensé par les petites écoles ou les couvents, il convient sans doute de rappeler le geste des missionnaires qui assumèrent souvent le rôle de maîtres ambulants pour dispenser, avec les bienfaits de leur ministère apostolique, les éléments du savoir humain. Et ils furent secondés dans leur tâche par des laïques et quelques notaires qui s'instituèrent pédagogues ${ }^{15}$. De plus les Frères Hospitaliers ou

$14 \mathrm{M}^{\mathrm{gr}}$ Amédée Gosselin, op. cit., p. 120; voir également les Registres des TroisRivières.

$15 \mathrm{M}^{\mathrm{gr}}$ Têtu, Les Evêques de Québec, Québec, 1889, p. 191. Voir également $\mathrm{M}^{\mathrm{gr}}$ Amédée Gosselin, op. cit., p. 127. 
Frères Charron assurèrent également la tenue de quelques écoles dans les agglomérations éloignées des centres ${ }^{16}$. Et l'on pourrait ajouter, à titre d'hypothèse très plausible - les témoignages précis faisant défaut sur ce point - que quelques curés occupèrent sans doute leurs loisirs à enseigner la lecture et l'écriture aux enfants de leur paroisse.

De toutes ces précisions, on ne peut évidemment conclure que l'instruction primaire des garçons fut très poussée dans les campagnes au XVII ${ }^{\bullet}$ et même au XVIII ${ }^{\circ}$ siècle; toutefois, les enquêtes faites dans les registres des paroisses et dans les greffes des notaires établissent qu'un très grand nombre de personnes savaient lire et écrire; d'autre part, les statistiques du Séminaire de Québec prouvent également qu'un groupe important d'élèves se présentaient chaque année prêts à commencer le latin; plusieurs venaient de la campagne ${ }^{17}$, preuve qu'ils avaient dû fréquenter l'école élémentaire de leur paroisse ou recevoir des leçons dans leur famille.

Que valaient ceux qui assumèrent la direction des petites écoles? Le problème du personnel enseignant ayant été résolu par l'Eglise, l'autorité religieuse s'occupa de très près du recrutement et de la formation des maîtres. Les Jésuites, les Récollets, les Sulpiciens, les Ursulines, les Sœurs de la Congrégation de Notre-Dame et les Frères Hospitaliers de Saint-Joseph de la Croix, dits Frères Charron s'adonnèrent à l'éducation de la jeunesse. Dans l'ensemble, la plupart de ces pédagogues n'ont d'autre diplôme que celui de leur bonne volonté et de leur dévouement à une cause excellente. Les autorités religieuses et civiles verront cependant à exiger des certificats de moralité et de compétence. D'autre part, le programme des petites écoles n'étant pas tellement compliqué, il était relativement facile aux maîtres de posséder les connaissances requises pour tenir convenablement leur classe, sinon pour enseigner selon toutes les exigences de la pédagogie moderne.

Un dernier mot sur la fréquentation scolaire. On peut bien se demander ce qu'elle fut, dans ce pays au climat rigoureux, où les moyens de communication faisaient grandement défaut, où les maîtres étaient rares, les distances à parcourir, considérables, la guerre, presque continuelle, où enfin les raisons ne manquaient pas pour excuser l'absence aux classes. Il ne faut donc pas s'attendre à une assiduité extraordinaire: nos ancêtres ont fait tout simplement leur possible et cela parfois jusqu'à l'héroïsme.

Voici plutôt des faits: la petite école dirigée par les Jésuites de Québec comptait, en 1699, au delà de cent élèves; le couvent des Ursulines qui eut des pensionnaires et des externes reçut, de 1640 à 1739 , environ mille deux cents pensionnaires, soit une moyenne de douze par année.

D'ailleurs, la situation n'était pas tellement différente en France à cette époque. Selon l'historien Taine, à la veille de la Révolution, il

16 L’Hôpital Général des Sœurs de la Charité (Montréal, 1916), pp. 33, 47, 56.

$17 \mathrm{M}^{\mathrm{gr}}$ Amédée Gosselin, op. cit., p. 136. 
existait dans la mère-patrie, aussi bien qu'au Canada, UNE école environ par deux paroisses et, dans plusieurs petits villages éloignés et sans ressources, le curé ou le vicaire était le seul personnage à savoir lire ou écrire. Serait-il exagéré de prétendre, qu'à certains points de vue, la situation fut meilleure dans la colonie où les registres démontrent péremptoirement qu'en toutes les parties de la Nouvelle-France on savait écrire et signer son nom. C'est que d'abord la famille avait fait sa part, la mère enseignant à ses enfants et la grande sœur aux plus jeunes. La petite école, pour modeste qu'elle fût, prépara une génération sachant au moins les rudiments de l'instruction ${ }^{18}$.

C'est au cardinal de Richelieu qu'il faut attribuer vraisemblablement la première idée de l'enseignement technique en France; on dut malheureusement attendre deux siècles avant de mettre à exécution, de façon sérieuse, des directions aussi sages. Au Canada cependant, les autorités civiles et religieuses se préoccupèrent, dès le milieu du $\mathrm{XVII}^{\circ}$ siècle, d'organiser un enseignement qui tiendrait le milieu entre les classes élémentaires et le cours classique. Les Jésuites, l'intendant Talon et $\mathbf{M}^{\mathbf{g r}}$ de Laval s'intéressèrent particulièrement à l'instruction des jeunes gens qui n'avaient pas la vocation à l'état ecclésiastique et qui ne pouvaient, d'autre part, aspirer aux carrières libérales. A leur intention, on fonda à Québec, un Ecole des Arts, à Saint-Joachim et à Montréal, une Ecole d'Arts et Métiers. En raison de la part considérable que prit $\mathrm{M}^{\mathrm{gr}}$ de Laval dans ces fondations, nous préférons traiter cette question dans la troisième partie de cette étude.

Quelle fut l'organisation de l'enseignement SECONDAIRE en Nouvelle-France au XVII ${ }^{\circ}$ siècle? Dans quelle mesure les institutions établies sur les bords du Saint-Laurent se rapprocheront-elles ou s'écarterontelles de celles de la mère-patrie? Voilà ce qu'il nous faut maintenant fixer.

La colonie naissante comptait à peine trois cents personnes, en 1635, que nous voyons les Jésuites fonder à Québec, à la demande des habitants, leur premier collège, où l'on enseigna d'abord les éléments du latin. Dès 1655, l'organisation du cours classique était complète: classes de grammaire, d'humanités, de rhétorique et de philosophie. Quelques années plus tard, à la demande de $\mathrm{M}^{\mathrm{gr}}$ de Laval, on commença même l'enseignement de la théologie. Et la fondation du séminaire de Québec, en 1668, ne nuisit en rien au Collège puisque cette institution ne fut, durant tout le régime français, qu'une maison de pension et de direction dont les élèves allaient prendre les leçons chez les Jésuites.

Le Collège des Jésuites fut donc le seul établissement d'enseignement secondaire complet au Canada, jusqu'à 1760. Pour tout dire, il faut mentionner aussi les écoles latines dont le but principal était de suppléer au collège, du moins durant les premières années du cours. Ces établissements étaient des séminaires presbytéraux comme il en surgit plusieurs à cette époque en Normandie. Les cinq principales fondations de ce genre furent établies à la Pointe-de-Lévy, à Saint-Joachim, au Château-

18 Taine, Origine de la France contemporaine, vol. VIII, p. 157. 
Richer et deux à Montréal où l'une fut tenue par les Sulpiciens et l'autre par les Jésuites ${ }^{19}$.

On peut difficilement imaginer que les Jésuites, fondateurs et organisateurs du Collège de Québec, formés eux-mêmes selon les méthodes et les disciplines en honneur dans leur Compagnie, aient adopté ici une formule différente de celle qu'ils avaient connue et pratiquée en France. $\mathrm{M}^{\mathrm{gr}}$ de Laval, le Père Germain et le Père de Rochemonteix ne pensent pas autrement; ce dernier écrivait à ce sujet que " le Collège de Québec était une reproduction en petit, mais complète des Collèges de France ${ }^{20}$ ".

Nous avons souligné toute l'importance donnée au latin, dans les Collèges des Jésuites, au XVII ${ }^{\mathrm{e}}$ siècle: il était interdit aux professeurs et aux élèves de parler français, seule la langue latine était permise; les notes des élèves étaient rédigées en latin et devaient donner un aperçu du travail, du talent et du succès de chacun. C'est à cette époque que les Messieurs de Port-Royal entreprirent la réforme du cours secondaire en préconisant l'enseignement systématique du français. Comme le Collège de Québec était alors à peine organisé, il est probable que le cours n'y fut jamais exclusivement latin.

Parmi les autres matières inscrites au programme, mentionnons le grec, la géographie, l'histoire et les sciences. Il semble bien toutefois que le cours de philosophie n'y fut que de deux ans au lieu de trois, comme c'était la pratique en France. Cet enseignement était complété par des séances dramatiques et littéraires pour souligner la visite de personnages officiels de passage au Collège.

Les manuels scolaires utilisés sont, à peu de chose près, les mêmes que ceux du Collège Laflèche: tels sont par exemple la Grammaire latine de Despautère, la Méthode facile pour décliner et conjuguer de Meslier, les Rudiments de la langue latine, du P. Codret, les ouvrages de Cicéron, Virgile, Sénèque, Ovide, Quinte-Curce, etc., les Préceptes de Rhétorique de Soarès, la Rhétorique du Père Jouvency, etc. Et voilà pour le latin. Quant au grec, nos jeunes étudiants s'y initiaient dans les ouvrages suivants: la Grammaire grecque de Nicolas Chénard, Institutionnes absolutissimae in linguam graecam (1617), Rudimenta linguae graecae du Père Gretseri (1656), Règle des accents et des esprits par le Père Labbé (1655), Grammaire grecque du Père Meslier (1702), etc. Enfin pour l'enseignement de la philosophie, on suivait Aristote et saint Thomas.

Il reste la fréquentation scolaire. A la fin du $\mathrm{XVII}^{\mathrm{e}}$ siècle, le Collège de Québec accueillait de cent à cent vingt élèves (externes et pensionnaires), sans compter les vingt-cinq ou trente pensionnaires qui logeaient au Petit Séminaire. Les autres institutions, celles de Montréal

19 La ville des Trois-Rivières paraît également posséder un collège tenue par les Récollets: voir à ce sujet Antoine Roy, Les lettres, les sciences et les arts au Canada sous le régime français, Paris, 1930, p. 20.

20 Le P. Germain au P. Assistant, 5 novembre 1711; Cf. Relations... Ed. Thwaites, vol. LXVI, p. 208. 
et les écoles latines accueillirent sans doute beaucoup moins d'élèves. Et tous, il faut l'ajouter, ne sont pas allés jusqu'à la fin du cours: cela s'explique facilement si l'on se rappelle les difficultés de l'époque, la guerre continuelle, les besoins de la terre et la pauvreté de nos premiers colons.

Et puis, souligne le chanoine Lionel Groulx, "que pouvait bien importer plus d'instruction classique à des familles de pauvres gens ou de médiocre aisance, vivant en un pays isolé, à peine développé, encore à l'écart des grandes rivalités commerciales et d'une vie politique réduite au minimum ? De plus, il faut tenir compte qu'à ceux-là qui étudient, seules ou à peu près, viennent s'offrir la carrière ecclésiastique et quelques rares fonctions administratives, en ce Canada d'ancien régime où les carrières libérales n'existent que peu ou point, les avocats n'y étant point tolérés, les médecins étant presque toujours d'importation française, et les notaires de cette lointaine époque n'ayant besoin ni de grammaire ni même d'orthographe ${ }^{21}$.D

L'organisation scolaire sous le régime français se compléta par une ébauche d'enseignement supérieur. En effet, l'enseignement des mathématiques était en grande faveur dans tous les collèges des Jésuites: à Québec cependant, le cours ne durait que deux ans, alors qu'il était de trois ans dans les Collèges de France. C'est dire que les mathématiques et l'hydrographie se donnaient à part, comme un enseignement particulier: le cours secondaire comportait lui-même des leçons dans cette matière, mais à un degré moins avancé.

Martin Boutet, arpenteur, semble bien avoir été le premier professeur de ces mathématiques avancées. Il enseigne dès 1665 , même si sa nomination officielle date de 1671 , alors qu'il inaugure une classe offcielle subventionnée par l'Etat. L'intendant Talon, en effet, rêvait d'établir ici une "Académie de marine " pour la formation de pilotes et d'arpenteurs.

L'un des plus illustres élèves de cette institution fut le découvreur du Mississipi, Louis Jolliet. Les successeurs de Martin Boutet à la chaire d'hydrographie furent, pour la plupart, des Pères Jésuites et c'est l'un d'eux qui précise que l'on entend par ce terme: "la description des eaux au point de vue géographique afin d'en déterminer la position sur le globe terrestre et au point de vue de la navigation afin d'en démontrer l'utilité ${ }^{22}$ ». Le Roi s'intéressa toujours de façon pratique (il payait le salaire du professeur) à cette Ecole où se formèrent des pilotes, des capitaines, des arpenteurs, des explorateurs et même des ingénieurs.

$\mathrm{Au}$ chapitre de l'enseignement supérieur ou mieux spécial, il convient de mentionner ici au moins, les cours de Théologie donnés en vue de préparer, pour la colonie naissante, un clergé indigène. Malheureusement, ce grand séminaire ne suffit pas à la tâche: en 1760, le clergé

21 Chan. L. Groulx, L'enseignement français au Canada, I, 25.

22 Rochemonteix, Un collège des Jésuites aux $X V I I^{e}$ et $X V I I I^{e}$ siècles, vol. IV, pp. 110 et 133 . 
tant séculier que régulier comprenait 163 personnes dont 51 seulement étaient nées au pays ${ }^{23}$.

Il n'y eut, sous le régime français, aucune organisation scolaire régulière et officielle, du moins dans le sens où nous l'entendons aujourd'hui. Les petites écoles donnaient aux fils de cultivateurs les rudiments de l'instruction primaire, le Collège de Québec préparait aux professions libérales, c'est-à-dire presque exclusivement à l'état ecclésiastique. L'éducation était plutôt une affaire de famille: nous chercherions en vain un code scolaire réglementant l'école avant 1760 . L'autorité suprême en matière d'éducation, c'est l'évêque. Et sur ce point, il recevra un appui sans réserve des autorités civiles, l'intendant, les gouverneurs et le Roi lui-même. Cet état de choses ne doit pas nous surprendre: le rôle de l'Etat, dans le champ de l'éducation, en France, au XVII ${ }^{\circ}$ siècle, consistait surtout à surveiller, à diriger et à régulariser. Ici, comme là-bas, le clergé fonda des écoles et le gouvernement l'encouragea de son autorité, de ses conseils et de ses deniers.

Que valaient ces écoles du XVII ${ }^{\mathrm{e}}$ siècle? Quels en furent les résultats? L'enseignement dut s'adapter aux besoins et aux ressources de la colonie. Les éducateurs de cette époque lointaine firent de louables efforts pour synchroniser leurs méthodes et leurs programmes avec ce qui se faisait alors dans les meilleurs collèges de France. La formation générale qu'en garda notre peuple se concrétisa dans une volonté ardente et déterminée de survivre lorsque vint le changement d'allégeance en 1760. C'est l'école qui a façonné l'élite de la colonie durant le XVII ${ }^{\circ}$ siècle et c'est elle encore qui la préparera aux épreuves que lui réserve le XVIII'.

L'organisation scolaire en France au $\mathrm{XVII}^{\mathbf{e}}$ siècle fut passablement différente de ce qu'elle est aujourd'hui; il en fut de même au Canada, où le clergé, les missionnaires et l'évêque furent les initiateurs dont l'action assura la création des écoles indispensables dans le secteur primaire aussi bien que sur le plan de l'enseignement secondaire. Il nous reste maintenant à montrer le rôle précis de $M^{\mathrm{gr}}$ de Laval dans ce domaine: c'est ce que nous ferons dans la troisième partie de ce travail.

\section{III - $\mathbf{M}^{\mathrm{gr}}$ DE LAVAL ET L'ÉDUCATION.}

Le zèle pour la diffusion des petites écoles, l'appui accordé à la communauté naissante des Sœurs de la Congrégation de Notre-Dame, la fondation d'écoles latines, l'initiative hardie du Cap-Tourmente, l'établissement du Grand Séminaire, puis du Petit Séminaire de Québec: voilà une énumération éloquente qui illustre mieux que de longues considérations tout le zèle de $\mathrm{M}^{\mathrm{gr}}$ de Laval pour assurer les progrès de l'éducation dans cet immense diocèse confié à ses soins.

Il n'est pas au Canada depuis dix ans que le roi Louis XIV lui écrit:

- Comme j'ai été informé du soin continuel que vous apportez pour bien vous acquitter des fonctions épiscopales et maintenir les peuples

2. Antoine Roy, op. cit., p. 22. 
dans leurs devoirs envers Dieu et envers moi, par la bonne éducation que vous donnez et faites donner aux enfants, je vous écris cette lettre pour vous témoigner le gré que je vous en sais et vous exhorter de continuer une conduite si bonne et si nécessaire ${ }^{24.0}$

Cette lettre était datée du 9 avril 1667. Le ministre Colbert avait écrit de son côté quelques jours plus tôt:

- Permettez-moi, Monsieur, de vous supplier, quoique vous fassiez l'une de vos plus importantes occupations de bien faire élever les enfants, d'en user toujours à leur égard avec la même bonté que vous avez fait jusqu'ici, parce qu'il est certain que c'est le meilleur moyen de bien policer la colonie et d'y former des gens capables de servir Dieu et le prince, dans toutes les professions différentes où ils se trouveront engagés pendant le cours de leur vie ${ }^{25}$.»

Mais voyons d'abord les initiatives de $\mathrm{M}^{\mathrm{gr}}$ de Laval pour la multiplication des petites écoles. La ville de Québec comptait environ 1,200 habitants en 1685: la partie la plus importante de la population était alors groupée à la basse-ville. L'état des chemins et la distance empêchaient bon nombre d'enfants de se rendre à la petite école tenue par les Jésuites. $\mathbf{M}^{\mathrm{gr}}$ de Laval songeait depuis longtemps à ouvrir une classe pour desservir cette partie de la cité. Après l'incendie de 1682, le vieux magasin de l'ancienne Compagnie des Cent-Associés n'avait pas été restauré et l'emplacement était disponible. Le gouverneur, M. de la Barre, le lui accorda par acte notarié au mois d'octobre 1683 et il semble probable que, malgré toutes sortes de difficultés, la maison d'école fut construite, en même temps que la chapelle, vers $1688{ }^{26}$.

La côte de Beaupré fut surtout l'objet des attentions et des générosités de $\mathrm{M}^{\mathrm{gr}}$ de Laval: les fondations du Cap-Tourmente et du ChâteauRicher méritent ici plus qu'une simple mention.

Quelque temps après la fondation du Petit Séminaire, $\mathrm{M}^{\mathrm{gr}}$ de Laval ouvrait, au Cap-Tourmente, une ferme modèle où les élèves vaquaient d'une part aux travaux de la terre, mais où également ils suivaient des leçons de catéchisme, de lecture, d'écriture et d'arithmétique. C'était ce que nous appellerions aujourd'hui une Ecole moyenne d'agriculture. Cette école se recruta de deux façons: les élèves y venaient directement des paroisses environnantes ou les autorités du Séminaire de Québec y dirigeaient ceux qui n'avaient pas d'aptitudes pour compléter leurs humanités. Il semble bien évident que cette école existait avant 1676 . En 1685 , le grand-vicaire, $\mathrm{M}^{\mathrm{gr}}$ de Saint-Vallier, faillit la transformer en collège classique mais, avec le retour de France de $\mathrm{M}^{\mathrm{gr}}$ de Laval, tout rentra dans l'ordre. En 1690, l'école était plus florissante que jamais: elle comptait alors 40 élèves " entretenus par leurs parents desquels on ne prend pour leur entretien que trois livres et un minot de sel par mois. On y en reçoit même quelques-uns par charité ${ }^{27}$.» Pour montrer son

24. $\mathrm{M}^{\mathrm{gr}}$ Amédée Gosselin, op. cit., p. 41.

$25 \mathrm{M}^{\mathrm{gr}}$ Amédée Gosselin, op. cit., p. 42.

26 Archives du Séminaire de Québec.

27 Etat des Revenus des Communautés établies en la Nouvelle-France avec les charges, anno 1690. Archives de la Marine, F. 3. 
attachement à cette œuvre, $\mathrm{M}^{\mathrm{gr}}$ l'Ancien - comme on l'appelait alors fondait, en 1693, six pensions pour des élèves pauvres:

"Ces enfants, est-il dit dans le contrat, doivent être du pays, de bonnes mœurs, propres au travail; ils seront choisis par les Supérieurs et Directeurs pour être nourris, entretenus et instruits aux bonnes mœurs, à la piété, à lire, à écrire ou formés au travail ou à quelques-uns des métiers qui s'y exercent jusqu'à ce qu'ils aient atteint l'âge de 18 ans, auquel âge ils sont capables de gagner leur vie, d'être à gages et de n'être plus à charge au Séminaire 28,.

Cette fondation du Cap-Tourmente ou de Saint-Joachim fut toujours une des œuvres de prédilection de $\mathrm{M}^{\mathrm{gr}}$ de Laval. Le 20 octobre 1699 Champigny écrivait au ministre: "L'ancien évêque vit saintement dans sa retraite, ne se mêlant que de la conduite de son séminaire de SaintJoachim. Cet établissement est considérable... On y élève et instruit quantité de jeunes gens, fils d'habitants et le tout se conduit avec beaucoup d'ordre et d'avantage pour la colonie ${ }^{29}$." La petite école de StJoachim vivra, semble-t-il, durant toute la domination française: mais, elle était bien loin; aussi voyons-nous les habitants de la Côte de Beaupré demander l'ouverture d'une autre petite école. $\mathrm{M}^{\mathrm{gr}}$ de Laval, seigneur de Beaupré, ne pouvait refuser cet avantage à ses censitaires. Aussi fit-il construire au Château-Richer, avant 1674, une maison destinée à l'instruction de la jeunesse. Lorsqu'il fit cession de ses biens au Séminaire, l'évêque de Pétrée parle ainsi de cette fondation:

- une maison appelée vulgairement le petit séminaire, située en la

paroisse et proche l'église Notre-Dame du Château-Richer... comme

l'ayant fait bâtir de neuf ${ }^{30}$.»

Il s'agit ici, tout simplement, d'une "petite école» destinée aux garçons comme l'indique bien un autre acte du 31 octobre 1687 alors que $\mathrm{M}^{\mathrm{gr}}$ de Laval et les Messieurs du Séminaire faisaient donation d'un arpent de terre à la fabrique, soulignant que le presbytère "y dessus construit 》servait de petite école.

Grâce au zèle de $\mathrm{M}^{\mathrm{gr}}$ de Laval, la paroisse du Château-Richer eut donc, très à bonne heure, sa petite école de garçons. Les principaux maîtres de cette école furent des laïques: Charles Roger que l'évêque de Québec amena probablement de France en 1675, Jacques-Eustache Rondeau, Toussaint Lafrance.

Le problème $d u$ recrutement $d u$ personnel enseignant masculin préoccupait l'Evêque de la Nouvelle-France: ainsi en 1685, un jeune homme se présente à lui avec l'intention de passer en Canada et d'y devenir prêtre. $\mathbf{M}^{\mathrm{gr}}$ de Laval, après l'avoir examiné, avisait le Séminaire qu'il ne l'avait " jugé propre qu'à tenir les petites écoles, en demeurant avec un ecclésiastique dont il aurait $\operatorname{soin}^{31}$ ». Cette décision ne manque

28 Génaple, not. Archives du Séminaire.

29 Rochemonteix, Les Jésuites..., vol. III, p. 352.

30 Archives du Séminaire. Cf. $\mathrm{M}^{\mathrm{gr}}$ Amédée Gosselin op. cit., p. 61.

31 Id. p. 63. Au témoignage de son biographe, l'abbé Auguste Gosselin, Mgr de Laval donna, en 1701, la somme de 8,000 francs pour que le Séminare mît à la ferme modèle de Saint-Joachim un maître qui enseignait aux trois élèves dont il avait fondé la pension * un commencement d'humanités afin qu'ils devinssent propres à être maîtres d'écoles.» Auguste Gosselin, Vie de $M^{g r} d e$ Laval, I: 570. 
pas d'intérêt dans l'histoire de nos premiers maîtres d'école: en effet, on en verra qui seront à la fois, bedeau, chantre, domestique du curé et maître d'école. Du reste, plusieurs notaires cumuleront, de leur côté diverses fonctions, celles de tabellion, de greffier, de chantre et de maître d'école. Dans les deux cas, lorsque le sujet était honnête, de bonnes mœurs et capable d'enseigner à lire et à écrire, il suffisait.

En 1702, cette école du Château-Richer devint une Ecole latine et même un pensionnat; ce dernier fut fermé en 1705 . $\mathrm{M}^{\mathrm{gr}}$ de Laval avait su inspirer à ses ouailles le goût de l'instruction: la maison d'école ayant besoin de réparations, nous voyons, en 1704, les habitants de toute la côte faire des quêtes afin d'aider le vénérable prélat "à mettre la maison seigneuriale en état d'y faire les petites écoles.»

C'est le lieu, me semble-t-il, de parler d'une fondation tout aussi importante, celle du couvent des Sœurs de la Congrégation de Notre-Dame au Château-Richer; cette fondation fut faite, à partir de 1693, par $\mathbf{M}^{\mathrm{gr}}$ de Laval et les Messieurs du Séminaire. Trois ans après le début des travaux, ces derniers avaient dépensé 10,000 livres pour l'établissement. L'évêque de Québec et son Séminaire complétèrent leur œuvre en s'engageant, le 23 février 1697, à payer chaque année la somme de 300 livres pour l'entretien de deux Sœurs:

- Ayant considéré que les petites écoles pour l'instruction et bonne éducation des filles de la dite seigneurie établies depuis quelques années en la paroisse du Château-Richer auraient peine à se soutenir s'il n'y était pourvu, d'une manière stable et solide à la subsistance des maîtresses d'école qui y ont été logées et entretenues jusqu'à présent par les soins et frais du dit seigneur ancien évêque et du Séminaire, poussés du désir de procurer la gloire de Dieu et le salut des âmes dans l'instruction et bonne éducation des filles des habitants de Beaupré, et voulant comme seigneur du dit lieu gratifier les dits habitants en procurant d'une manière permanente et assurée autant qu'il est en eux aux dites filles les moyens de les instruire et bien élever, comme étant la dite instruction et bonne éducation une chose des plus importantes au bien des familles... $\quad M^{g r}$ de Laval et le Séminaire s'engagent a payer, chaque année, 300 livres monnaie de ce pays, payables en bons effets pour contribuer à la nourriture et entretien de deux maîtresses d'école jugées capables et de bonnes mœurs qui seront au choix du dit Séminaire... ${ }^{32}$.)

Le zèle de $\mathrm{M}^{\mathrm{gr}}$ de Laval ne se refroidira pas. On le voit, en 1699 , rivaliser avec $\mathbf{M}^{\mathrm{gr}}$ de Saint-Vallier pour promouvoir l'instruction sur toute la côte de Beaupré. Pendant que ce dernier paie la pension de trois

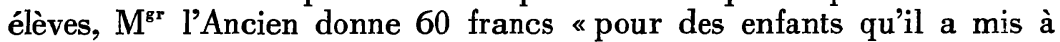
l'instruction chez les Sœurs du Château »; l'année suivante, il doublait ses générosités au profit des sept élèves.

La fondation d'une communauté religieuse enseignante de femmes reste un événement d'importance en ces débuts de la colonie. En 1657, Marguerite Bourgeoys ouvre sa première école dans une étable de pierres que M. de Maisonneuve avait mise à sa disposition. Le 20 mai 1669, $\mathbf{M}^{\mathrm{gr}}$ de Laval donnait à la fondatrice la permission d'instruire les enfants dans toute l'étendue de son diocèse; en 1671, le Roi approuvait la nou- 
velle communauté; enfin le 6 août 1676, l'évêque de Québec déclarait dans un mandement:

* sachant qu'un des plus grands biens que nous puissions procurer à notre église et le moyen le plus efficace pour conserver et augmenter la piété dans les familles chrétiennes est l'instruction et la bonne éducation des enfants; connaissant d'ailleurs la bénédiction que Notre-Seigneur a donnée jusqu'à présent à la dite Sœur Bourgeoys et à ses compagnes dans les dites fonctions des petites écoles où nous les aurions employées et voulant favoriser leur zèle et contribuer de tout notre pouvoir à leur pieux dessein, Nous avons agréé et agréons l'établissement de la dite Bourgeoys et des filles qui se sont unies avec elle ou qui y seront admises à l'avenir leur permettant de vivre en communauté... ${ }^{33}$."

$\mathrm{M}^{\mathrm{gr}}$ de Laval n'attendit pas cette approbation officielle pour faire appel au dévouement des Soeurs de la Congrégation de Notre-Dame: dans un rapport au Saint-Siège, il déclare que, dès 1664 - et peut-être avant - il a envoyé des maîtresses d'écoles assez âgées pour prendre la direction des écoles de filles aux Trois-Rivières. Il s'agit ici, sans aucun doute, des Sœurs de Marguerite Bourgeoys; l'on sait, d'autre part, que la Sœur Raisin et une compagne furent les premières à y tenir école, probablement une école mixte.

Les petites écoles ne furent pas les seules à bénéficier du dévouement de l'Evêque de Québec: il fut l'instigateur de l'enseignement professionnel par la création d'Ecoles d'Arts et Métiers.

Dès $1668, \mathrm{M}^{\mathrm{gr}}$ de Laval était propriétaire de deux fermes considérables au Cap-Tourmente, fermes sur lesquelles il voulait ouvrir une école élémentaire, une école latine, une ferme modèle et un établissement pour les arts et métiers. Sur l'une de ces fermes, la ferme d'en haut ou grande ferme, se trouvait érigée une maison à deux étages dans laquelle on pouvait loger bon nombre d'enfants. $M^{\mathrm{gr}}$ de Laval comprit immédiatement le parti qu'il en pourrait tirer pour ces jeunes gens dont les goûts et les aptitudes semblaient se tourner vers l'apprentissage intensif d'un métier indispensable dans une colonie naissante. Revenu d'un voyage en France en 1688, le prélat résolut de donner une vigoureuse impulsion à cet établissement. Il commença par " rassembler un bon nombre de jeunes gens, la plupart de la campagne, pour les appliquer... à des travaux dans lesquels ils montraient une grande dextérité ${ }^{34}{ }^{\text {» }}$. A leur intention, il établit des bourses d'études, «des pensions » comme on disait alors: les candidats devaient être du pays, de bonnes mœurs, propres au travail; en retour de leur application, ils étaient nourris, logés et formés à quelqu'un des métiers inscrits au programme de l'école. Un mémoire de 1685 nous renseigne sur ces métiers de l'Ecole de SaintJoachim: la menuiserie, la sculpture, la peinture, la dorure; de plus, on trouve encore dans cette institution, des tailleurs, des cordonniers, des taillandiers, des serruriers, et des couvreurs qui enseignent leur métier aux jeunes gens.

Cette école connut une belle prospérité à la fin du XVII ${ }^{e}$ siècle. L'abbé Louis Soumande en est alors le directeur: c'est un homme

33 Mandements des Evêques de Québec, I, p. 100.

34 L'Abeille, I, No 41. 
méthodique et généreux qui attire les élèves par la fondation de nombreuses bourses d'études; le directeur artistique est Jacques Leblond dit Latour qui arrive en 1690 et les autres professeurs Denys Mallet, Claude Baillif et François de Lajoue ${ }^{\mathbf{3 5}}$.

Enfin le petit séminaire de Québec fut, durant tout le régime français, une simple maison de pension pour les élèves qui poursuivaient leurs études régulières au Collège des Jésuites. $M^{\mathrm{gr}}$ de Laval décida cependant d'y faire donner des cours sur quelques-uns des métiers les plus utiles: c'est ainsi que l'on y relève ceux de menuisier, de charpentier, de couvreur, de cordonnier, de couturier, de maçon, etc. Il est donc bien évident que Québec posséda, du moins durant quelques années, une Ecole d'Arts et Métiers identique à celle de Saint-Joachim. Cependant le principal art qui fut cultivé à cette école fut la sculpture.

Cette fondation de $\mathrm{M}^{\mathrm{gr}}$ de Laval disparut, semble-t-il, vers 1730: à partir de 1705, la succursale de Saint-Joachim (en réalité, l'école de Saint-Joachim et celle du Petit Séminaire de Québec ne sont qu'une seule et même école: les professeurs sont identiques et les élèves se déplacent au besoin) devient plutôt une école d'agriculture et, après la mort de l'abbé Soumande en 1715, elle ne bat plus que d'une aile; elle est trop éloignée de Québec et les artisans urbains ont un urgent besoin d'apprentis.

Cette initiative hardie de $M^{\mathrm{gr}}$ de Laval est une nouvelle preuve de son sens pratique et de sa générosité, comme aussi du zèle du Séminaire de Québec; les Frères Charron tenteront une entreprise similaire à Montréal.

Il nous reste à montrer la sollicitude de $\mathrm{M}^{\mathrm{gr}}$ de Laval pour l'enseignement secondaire et surtout à souligner la part qu'il prit dans la fondation du Séminaire de Québec.

A son arrivée en Nouvelle-France, en 1659, le premier évêque de Québec trouva le Collège des Jésuites en plein essor puisqu'il pouvait écrire au Saint-Siège quelques années plus tard: "A Québec, les Pères Jésuites ont un collège où les classes d'humanités sont florissantes et où les enfants vivent et sont élevés de la même manière qu'en France ${ }^{36}$." Mais le nouveau pasteur se rendit compte immédiatement de l'urgente nécessité de préparer des prêtres pour la colonie naissante: il demanda donc aux Jésuites d'assumer cette nouvelle tâche:

- A son arrivée, écrivait le Père Lalemant en $1670, \mathrm{M}^{\mathrm{gr}}$ de Laval cherchant des prêtres pour former un clergé et pour desservir les paroisses, et voyant qu'il ne pouvait en obtenir de France, jeta les yeux sur nos scholastiques indigènes qui, leurs lettres terminées, aspiraient à la philosophie. Comme il n'y avait personne autre que nous, nous entreprîmes l'œuvre, et en conséquence, ouvrîmes une classe de mathématiques et de théologie morale et scholastique, en sorte qu'il y en a cinq ou six d'entre eux qui sont promus aux ordres majeurs. $\mathrm{M}^{\mathrm{gr}}$ en a douze ou treize qui fréquentent nos classes, sans compter alios nostros convictores, c'est-à-dire nos autres pensionnaires qui aspirent à ces études ${ }^{37}$.» 
A quelle date fut inauguré ce cours de Théologie? Sûrement avant 1666, puisque, dès cette année-là, l'évêque en parle avec détails dans son rapport au Souverain Pontife ${ }^{38}$. Par ailleurs, dans un mandement publié à Paris, le 26 mars 1663, il établissait formellement un GRAND SEMINAIRE:

- On y élèvera et formera, disait-il, les jeunes clercs qui paraîtront propres au service de Dieu et auxquels, à cette fin, l'on enseignera la manière de bien administrer les sacrements, la méthode de catéchiser et prêcher apostoliquement, la théologie morale, les cérémonies, le plainchant grégorien et autres choses appartenant aux devoirs d'un bon ecclésiastique ${ }^{39}$."

Quelques années plus tard et après l'ouverture du Petit Séminaire (1677), $\mathbf{M}^{\mathrm{gr}}$ de Laval songea à construire son Grand Séminaire. Au mois de mai 1678 et avant de partir pour l'Europe, il en posait la première pierre. Les travaux furent poussés vivement en son absence; lorsqu'il revint, en octobre 1680, l'édifice était en bonne voie de parachèvement si bien que les ecclésiastiques purent y entrer en 1681. Les travaux cependant continuèrent jusqu'en 1698. C'était le grand corps de logis qui fait face au jardin et au fleuve: il se joignait à angle droit au Petit Séminaire (Cf. Gosselin, Auguste, II: 220.)

Mais ce grand Séminaire, il fallait l'alimenter, il fallait lui préparer des sujets. Le Collège des Jésuites était bien là, et il semble que $\mathbf{M}^{\mathrm{gr}}$ de Laval s'en serait peut-être contenté en ces premières années d'épiscopat si une lettre de Colbert, au printemps de 1668, ne fût venue presser l'exécution des plans de l'évêque. En effet, le ministre conjurait $\mathbf{M}^{\mathrm{gr}}$ de Laval de mettre tout en œuvre pour franciser les sauvages et le moyen tout désigné pour arriver à cette fin, c'était évidemment de mettre les sauvages en contact suivi avec les Français. A cette fin, $M^{\mathrm{gr}} \mathrm{l}^{\prime}$ Evêque de Québec décida de fonder un Petit Séminaire dont il fit l'ouverture offcielle, le 9 octobre 1668, jour de la Saint-Denys. Les treize premiers élèves, sept Français et six sauvages furent donc installés dans la maison que $\mathbf{M}^{\mathrm{gr}}$ de Laval avait achetée, deux ans plus tôt, de Guillemette Hébert, veuve de Guillaume Couillard. La francisation des sauvages ne fut pas un succès: un seul des six premiers sujets demeura au Séminaire environ cinq ans; quant aux autres, ils eurent bientôt la nostalgie des espaces illimités et de la vie de grande liberté dans les forêts: ils quittèrent vite le Petit Séminaire ${ }^{40}$.

En dépit de cet échec, l'effectif de la maison s'accrut rapidement: de 13 qu'il était en 1668, il passa à 32 en 1675-76; la maison étant devenue trop exiguë, $\mathrm{M}^{\mathrm{gr}}$ de Laval dut songer à une nouvelle construction. Le 24 avril 1675 , il avait justement échangé avec M. Berthelot, l'île d'Orléans pour l'île Jésus, et il en avait obtenu 25,000 francs de retour ! C'était une chance inespérée: il fit commencer les travaux sans retard sur cet emplacement situé à angle droit avec la cathédrale et attenant au chevet de l'église. Il y consacra également une partie des 6,000 livres

38 Mandements... I, p. 36.

39 Mandements des Evêques de Québec, I, p. 45.

40 Abbé Auguste Gosselin, Vie de $M^{g r}$ de Laval, 1890, I: 557-564. 
qu'il recevait annuellement du Roi pour les besoins de son Eglise. A la fin de l'année 1677, la maison était prête à recevoir des élèves. L'inauguration en fut faite le 7 décembre 1677 par $M^{\text {gr }}$ de Laval lui-même qui prononça le sermon de circonstance et "le lendemain, disent les Archives du Séminaire, fête de la Conception Immaculée de la Ste-Vierge, les enfants du Séminaire, après avoir fait leur rénovation, s'être confessés et avoir communié, commencèrent à s'y loger ». Ils étaient quinze sous la direction de Monsieur de Maizerets qui remplissait toujours les fonctions de directeur des élèves.

Comme on vient de le voir, le Petit Séminaire, c'est l'œuvre par excellence de $\mathbf{M}^{\text {gr }}$ de Laval. "Jusqu'en 1680, écrit le cardinal Taschereau dans son histoire manuscrite de la maison, le Séminaire n'eut d'autres ressources certaines que de faibles rentes, quelques terres achetées depuis peu, le désintéressement de ses membres et la bienveillance de son charitable bienfaiteur.» C'est alors que l'évêque de Québec, comprenant la précarité de la situation décida de donner tous ses biens au Séminaire, ne se réservant que l'usufruit jusqu'à sa mort. On se rappelle, d'autre part, que dès son arrivée à Québec, le prélat avait payé lui-même la pension de quelques élèves qui fréquentaient le collège des Jésuites; on se souvient également de sa générosité lors de la fondation du Petit Séminaire. Ce fut l'occasion pour $\mathrm{M}^{\mathrm{gr}}$ de Laval de créer des bourses à l'intention de ces enfants pauvres qui se destinaient à l'état ecclésiastique:

- Le Séminaire devra, dit-il dans l'acte, nourrir, loger, entretenir et élever aux études jusqu'à la fin de la théologie, huit pauvres enfants de bonnes mœurs, qui auront la vocation à l'état ecclésiastique, qui seront choisis par les directeurs ${ }^{41}$...

Ces largesses de $\mathrm{M}^{\mathrm{gr}}$ de Laval étaient providentielles car l'effectif du Séminaire continuait d'augmenter: en 1681, on atteignait quarante élèves, ce qui n'était pas de nature à réjouir le procureur, M. Dudouyt qui avait alors à entretenir et à nourrir cent vingt personnes à la maison !

C'est en 1693 que l'on commença la construction de la chapelle, tout en agrandissant les locaux qui servaient au Petit Séminaire: vers 1699 , tout fut terminé et $\mathrm{M}^{\mathrm{gr}}$ de Laval pouvait se réjouir des progrès incontestables de son œuvre et songer même à un repos bien mérité. Mais l'heure de l'épreuve allait sonner pour le vénérable prélat: le 15 novembre 1701, un premier incendie détruisait une bonne partie de l'œuvre édifiée au prix de tant de sacrifices: "J'estime le dommage de cette maison 30,000 livres pour son rétablissement seulement," écrivait Levasseur de Nérée. Sans se laisser abattre, $\mathrm{M}^{\mathrm{gr}}$ de Laval et les Messieurs du Séminaire décidèrent la restauration, forts de la sympathie générale et de l'appui de MM. Callières et Champigny qui s'empressèrent de solliciter les secours du roi.

Le désastre était à peine réparé que, de nouveau, le $1^{\text {er }}$ octobre 1705 , un nouvel incendie détruisit le Petit Séminaire de fond en comble. Gardant leur sérénité et leur confiance en la Providence, $\mathbf{M}^{\mathrm{gr}}$ de Laval

$41 \mathrm{M}^{\mathrm{gr}}$ Amédée Gosselin, op. cit., p. 394. 
et les directeurs du Séminaire entreprirent encore une fois la reconstruction. Cette foi et cette ténacité furent récompensées et serviront merveilleusement la cause de l'éducation secondaire au milieu du XVIII ${ }^{\circ}$ siècle: lorsque le Collège des Jésuites devra fermer ses portes, après la conquête, le Petit Séminaire de Québec sera là pour prendre la relève. Par delà les siècles, ce sera toujours l'œuvre du premier évêque de Québec qui perpétuera le rayonnement de la culture française sur le fier promontoire de Québec.

\section{CONCLUSIONS}

L'éducation en Nouvelle-France au $\mathrm{XVII}^{\mathrm{e}}$ siècle fut, avant tout, l'œuvre du clergé et des communautés religieuses. C'est toujours pour l'historien un sujet de profond étonnement de constater que, dans cette colonie qui vient à peine de naître, alors que toutes les énergies sont tendues vers la lutte contre la dureté de ce pays neuf et contre les incursions sans cesse renaissante des sauvages hostiles, c'est un sujet d'étonnement et d'admiration dis-je, que de constater cette préoccupation constante pour les valeurs spirituelles et pour la permanence, sur les bords du Saint-Laurent, de la pensée et de la culture française.

Ce zèle pour la fondation des petites écoles et pour l'établissement du Collège de Québec s'intensifiera encore à l'arrivée de $\mathbf{M}^{\text {gr }}$ de Laval: le premier évêque de Québec aura toujours présentes à l'esprit les paroles impératives du Maître à ses disciples assemblés pour un dernier adieu: "Allez, enseignez toutes les nations." Et ce devoir du pontife ne s'arrête pas qu'aux seules vérités surnaturelles: il a mission de faire connaître à tous sans exception, le Vrai, le Bien et le Beau, objets de l'attention et des recherches des grands esprits de tous les temps. A tous égards, François de Montmorency-Laval, premier évêque de Québec, fut véritablement le "père de la patrie canadienne».

25 septembre 1958.

Dr Louis-Philippe AudeT, de la Société royale du Canada 